\title{
All three components of the neuronal SNARE complex contribute to secretory vesicle docking
}

\author{
Yao Wu, ${ }^{1,2}$ Yiwen Gu, ${ }^{1,2}$ Mary K. Morphew, ${ }^{3}$ Jun Yao, ${ }^{1,2}$ Felix L. Yeh, ${ }^{1,2}$ Min Dong, ${ }^{1,2}$ and Edwin R. Chapman ${ }^{1,2}$ \\ 'Howard Hughes Medical Institute and 2Department of Neuroscience, University of Wisconsin, Madison, WI 53706 \\ ${ }^{3}$ Boulder Laboratory for 3D Electron Microscopy of Cells, Department of Molecular, Cellular, and Developmental Biology, University of Colorado, Boulder, CO 80309
}

B efore exocytosis, vesicles must first become docked to the plasma membrane. The SNARE complex was originally hypothesized to mediate both the docking and fusion steps in the secretory pathway, but previous electron microscopy (EM) studies indicated that the vesicular SNARE protein synaptobrevin (syb) was dispensable for docking. In this paper, we studied the function of syb in the docking of large dense-core vesicles (LDCVs) in live $\mathrm{PC} 12$ cells using total internal reflection fluorescence microscopy. Cleavage of syb by a clostridial neurotoxin resulted in significant defects in vesicle docking in unfixed cells; these results were confirmed via EM using cells that were prepared using high-pressure freezing. The membranedistal portion of its SNARE motif was critical for docking, whereas deletion of a membrane-proximal segment had little effect on docking but diminished fusion. Because docking was also inhibited by toxin-mediated cleavage of the target membrane SNAREs syntaxin and SNAP-25, syb might attach LDCVs to the plasma membrane through $\mathrm{N}$-terminal assembly of trans-SNARE pairs.

\section{Introduction}

The release of neurotransmitters and hormones from neurons and neuroendocrine cells involves a multistep pathway that culminates in the $\mathrm{Ca}^{2+}$-triggered fusion of secretory vesicles with the plasma membrane. A crucial step in this pathway concerns docking reactions that draw vesicles into contact with the plasma membrane before fusion (Verhage and Sørensen, 2008). Upon association with the target membrane, docked vesicles are thought to undergo priming reactions that make them competent to fuse; alternatively, priming might correspond to the docking reaction itself (Hammarlund et al., 2007). Once docked and primed, a rise in intracellular $\left[\mathrm{Ca}^{2+}\right]$ can then trigger membrane fusion and exocytosis.

At present, the molecular mechanisms that underlie the docking of secretory vesicles remain poorly understood. In one compelling hypothesis, SNARE proteins were proposed to mediate both docking and fusion of vesicles (Söllner et al., 1993). The v-SNARE synaptobrevin (syb) interacts with the t-SNAREs

Y. Wu and Y. Gu contributed equally to this paper.

Correspondence to Edwin R. Chapman: chapman@wisc.edu

M. Dong's present address is Dept. of Microbiology and Immunology and Division of Neuroscience, New England Primate Research Center, Harvard Medical School, Southborough, MA 01772.

Abbreviations used in this paper: BoNT, botulinum neurotoxin; HPF, highpressure freezing; LC, light chain; LDCV, large dense-core vesicle; SV, synaptic vesicle; TIRFM, total internal reflection fluorescence microscopy; $\mathrm{PAA}$, tissue plasminogen activator. syntaxin (syx) and SNAP-25 (25-kD synaptosomal-associated protein) to form trans-SNARE complexes (Rothman, 1994; Sutton et al., 1998; Weber et al., 1998). The core region of the SNARE complex is a parallel four-helix bundle formed by two $\alpha$ helices from SNAP-25 together with one $\alpha$ helix from both syx and syb. The associating segments are 60-70 aa long and are termed SNARE motifs. The SNARE complex has been proposed to assemble like a zipper, starting from the membranedistal $\mathrm{N}$ termini and progressing toward the membrane-proximal $\mathrm{C}$ termini of the SNARE motifs, with reports that argue both for and against this model (Han and Jackson, 2006; Sørensen et al., 2006).

It is well established that the SNARE complex corresponds to the minimal machinery for membrane fusion in eukaryotic cells (Weber et al., 1998). However, numerous EM studies had ruled out a role for SNAREs in docking, and this aspect of the SNARE hypothesis appeared to be untenable (Hunt et al., 1994; Broadie et al., 1995; O'Connor et al., 1997; Sørensen et al., 2003; Borisovska et al., 2005). Then, surprisingly, a handful of recent EM studies concluded that t-SNAREs are in fact involved in secretory vesicle docking in at least some cell

(c) 2012 Wu et al. This article is distributed under the terms of an Attribution-NoncommercialShare Alike-No Mirror Sites license for the first six months after the publication date (see http://www.rupress.org/terms). After six months it is available under a Creative Commons License (Attribution-Noncommercial-Share Alike 3.0 Unported license, as described at http://creativecommons.org/licenses/by-nc-sa/3.0/). 
types (de Wit et al., 2006; Hammarlund et al., 2007, 2008; de Wit et al., 2009). More specifically, syx and SNAP-25 were reported to form a plasma membrane complex for docking of large dense-core vesicles (LDCVs) in chromaffin cells (de Wit et al., 2006, 2009), and syx was reported to dock synaptic vesicles (SVs; Hammarlund et al., 2007; but see de Wit et al. [2006]) and LDCVs (Hammarlund et al., 2008) in neurons.

These new findings raise the question as to the identity of vesicle proteins that participate in docking. There are two SV proteins that have emerged as compelling candidates: syb and synaptotagmin I (syt-I). The $\mathrm{Ca}^{2+}$ sensor for rapid exocytosis, syt-I, binds directly to t-SNAREs (Chapman, 2008) and was reported to play a critical role in SV docking at the Drosophila melanogaster neuromuscular junction (Reist et al., 1998) andmore recently - in LDCV docking in chromaffin cells (de Wit et al., 2009). Moreover, a recent EM tomography study of presynaptic boutons from cultured hippocampal neurons from syt-I knockout mice revealed a marked decrease in docking of SVs; however, this effect was largely secondary as to decrease in the total number of vesicles (Liu et al., 2009), and another group reported no change in presynaptic ultrastructure (Geppert et al., 1994). A clear consensus regarding the contribution of syt-I to docking reactions, as measured by EM, has yet to emerge.

The other candidate docking protein, syb, had been ruled out by a plethora of earlier EM studies (Hunt et al., 1994; Broadie et al., 1995; Deák et al., 2004; Borisovska et al., 2005). However, unlike the case of t-SNAREs, recent studies continue to support the conclusion that syb is dispensable for docking of SVs and LDCVs (Gerber et al., 2008; Fernández-Busnadiego et al., 2010). If syb does not participate in the docking step, docking cannot be mediated by trans-SNARE complexes, as originally envisioned (Söllner et al., 1993).

A limitation of EM studies concerns the fixation and sample preparation methods that are used; these can introduce significant artifacts in membrane structure and cell morphology, which in turn can alter the distribution of vesicles. In addition, different distance criteria have been used to define docking via EM (ranging from 0 to $30 \mathrm{~nm}$ ), potentially contributing to some of the ambiguity among these studies. New insights regarding the function of syx and SNAP-25 in docking have been gained by using newer high-pressure freezing (HPF) fixation techniques that apparently reduce artifacts (Hammarlund et al., 2007). However, it remains unclear as to whether these artifacts can be fully eliminated by improvements in sample preparation for EM analysis. To investigate this issue, we performed EM using samples prepared using both chemical fixation and HPF methods. Moreover, we studied the docking of LDCVs in live PC12 cells using total internal reflection fluorescence microscopy (TIRFM; Axelrod, 1981, 2001). TIRFM enables visualization of the vesicles nearby the plasma membrane using an evanescent wave and has been used to study vesicle docking (Toonen et al., 2006) and fusion (Steyer et al., 1997; Zenisek et al., 2000).

Surprisingly, docking was strongly reduced upon cleavage of any of the three components of the neuronal SNARE complex with botulinum neurotoxins (BoNTs). Structure-function analysis of syb resulted in mutants that uncouple docking from fusion and supports the zipper model for SNARE complex assembly.

\section{Results and discussion}

Cleavage of syb by BoNT/D results

in LDCV docking defects

Our first objective was to determine whether syb plays a role in the docking of LDCVs in PC12 cells. To this end, we reduced the amount of functional syb by expressing the light chain (LC) of BoNT/D; this neurotoxin selectively cleaves rat syb I and II (Schiavo et al., 2000). Transfected cells were singled out for analysis via an in-vector marker, DsRed; $\sim 30-40 \%$ of cells were DsRed/BoNT/D-LC positive (unpublished data). Immunoblot analysis revealed a $\sim 40 \%$ reduction in the total amount of syb (Fig. 1 A). Thus, syb was efficiently cleaved in transfected cells, as further indicated by the lack of syb immunofluorescence (Fig. 1 B). Cleavage of syb resulted in the elimination of LDCV exocytosis triggered by high $\left[\mathrm{K}^{+}\right]$ (control, $24.8 \pm 5.2 / \mathrm{min} ;$ BoNT/D, $0.8 \pm 0.5 / \mathrm{min} ; \mathrm{P}<0.001$; Fig. 1 D), consistent with the effects of BoNT/D on secretion (Schiavo et al., 1993).

To study docking of LDCVs via TIRFM, a GFP-tagged cargo protein, tissue plasminogen activator (tPA)-GFP, was expressed as a vesicle marker (Tsuboi et al., 2004). The density of LDCVs near the plasma membrane was calculated from the number of GFP fluorescence puncta divided by area of the cell footprint (Fig. 1, E-G). Strikingly, a significant reduction ( $\sim 45 \%)$ in the density of docked LDCVs was observed when syb had been cleaved, as compared with control cells (control, $0.63 \pm 0.04 / \mu \mathrm{m}^{2} ;$ BoNT/D, $0.35 \pm 0.03 / \mu \mathrm{m}^{2} ; \mathrm{P}<0.001 ;$ Fig. $1, \mathrm{~F}$, $\mathrm{H}$, and $\mathrm{I})$, suggesting that syb participates in the docking step. We note that although the densities of docked LDCVs (per $\mu \mathrm{m}^{2}$ ) varied considerably from cell to cell, the distribution of docked vesicles across a population of cells (within a given condition) was comparable, as long as the number of cells used for each experiment was sufficiently large (Fig. S1).

We also analyzed the dynamics of LDCV docking by defining a docked vesicle as being present, for $5 \mathrm{~s}$, in the evanescent field (as detailed in Materials and methods). By these criteria, most vesicles were immobile (control, $\sim 80 \%, n=36$; BoNT/D, $\sim 70 \%, n=32$ ), and a significant reduction in LDCV docking density $(\sim 60 \%)$ - similar to the aforementioned static results-was observed when syb had been cleaved, as compared with control cells (control, $0.48 \pm 0.03 / \mu \mathrm{m}^{2}$; BoNT/D, $\left.0.20 \pm 0.03 / \mu \mathrm{m}^{2} ; \mathrm{P}<0.001\right)$.

In these experiments, the evanescent field decay constant, d, was $\sim 110 \mathrm{~nm}$, so the fluorescence intensity drops to $\sim 5 \%$ when $\mathrm{Z}=330 \mathrm{~nm}$. Hence, the signals reported here are from vesicles that lie within $330 \mathrm{~nm}$ of the plasma membrane. As shown in a previous study (Toonen et al., 2006), changes in the total number of vesicles within an $\sim 360$-nm range reliably reflect alterations in vesicle docking. Interestingly, Toonen et al. (2006) also reported that cleavage of syb by the LC of tetanus neurotoxin did not inhibit docking of LDCVs in chromaffin cells. However, the total number of LDCVs increased significantly $(>50 \%)$ in cells expressing the tetanus toxin LC, whereas the number of docked LDCVs did not change. These results indicate that the ratio of docked to total vesicles decreased, which is consistent with our observations. 


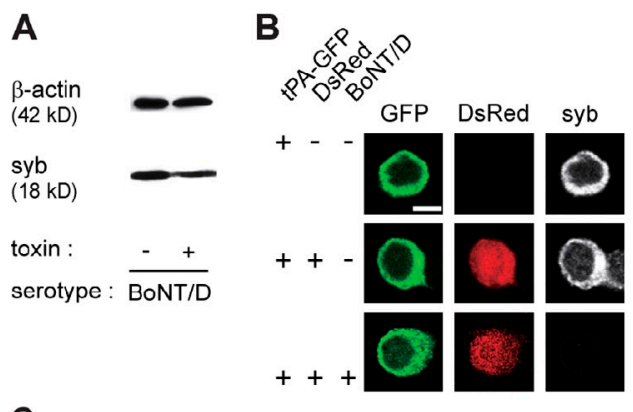

E

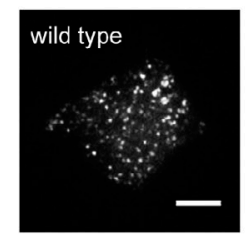

$\mathbf{F}$
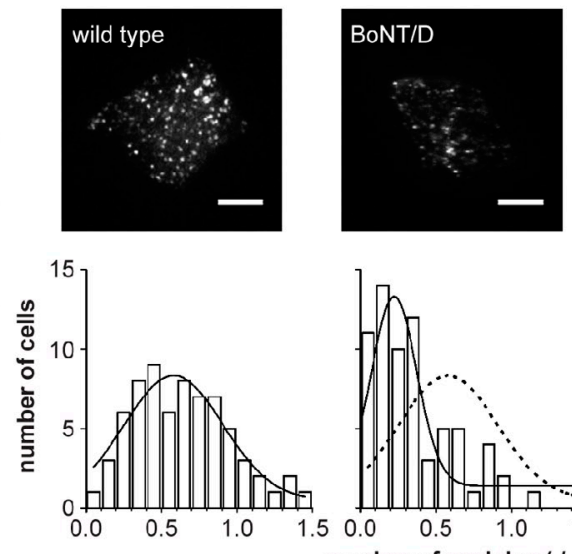

number of vesicles $\left(/ \mu \mathrm{m}^{2}\right)$
G
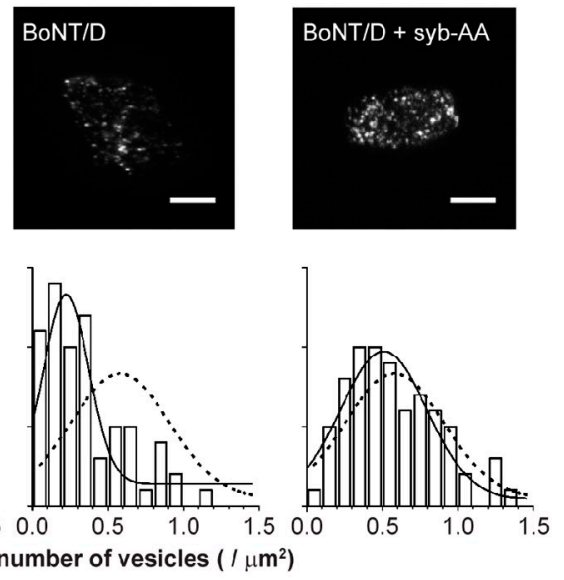

I

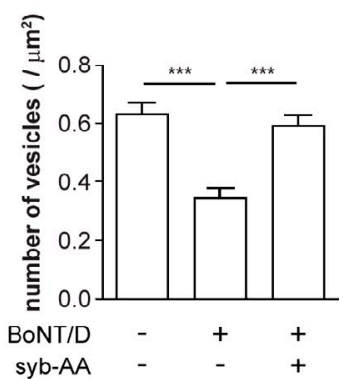

Figure 1. Cleavage of endogenous syb by BoNT/D reduces LDCV docking in PC12 cells. (A) Immunoblot analysis demonstrating cleavage of syb by BoNT/D. (B) Immunocytochemistry showing that syb (white) was detected in two control groups (tPA-GFP only; tPA-GFP plus the DsRed vector lacking the BoNT/D-LC, denoted DsRed) but not in cells expressing the LC of BoNT/D (DsRed vector that also expressed the BoNT/D-LC, denoted BoNT/D). (C) Immunocytochemistry showing that syb (white, right column) was always detected in PC12 cells coexpressing syb-AA, BoNT/D (red, middle column), and PAA-GFP (green, left column) but never in cells lacking syb-AA. Thus, the selected PC 12 cells that expressed BoNT/D-LC were most likely transfected with syb-AA in the TIRF experiments. (B and C) Bars, $5 \mu \mathrm{m}$. (D) Bar graph summarizing the rates of vesicle fusion triggered by high [ $\left.\mathrm{K}^{+}\right]$. Fusion was eliminated in syb-cleaved cells and was rescued by expression of syb-AA ( $n=4$ for all conditions, including untreated control cells). (E-G) Exemplary TIRF images (top images) and quantification of the density of docked vesicles (bottom images) in wild-type PC 12 cells and cells expressing BoNT/D with or without coexpression of syb-AA. Bars, $5 \mu \mathrm{m}$. Note that data obtained using untreated wild-type cells (E, bottom) were fit with a normal distribution curve (solid line); this curve is included in $F$ and $G$ as dashed lines. Data from three independent trials were summed and are plotted in each histogram. (H) Normalized cumulative distribution of LDCVs as a function of the density of LDCVs. (I) Bar graph showing the density of docked LDCVs in PC12 cells. (D-I) Wild-type control cells, $n=69$; BoNT/D, $n=68$; syb-AA, $n=74$. ${ }^{* * *}, \mathrm{P}<0.001$. Error bars represent SEM.

Nevertheless, there was still a lingering concern that the TIRFM measurements, with a 330-nm depth of field, reported changes in tethering (defined as an attachment with the vesicles further away from the plasma membrane than docked vesicles) rather than docking. EM has sufficient spatial resolution to address this issue, but, as previously noted, this method has led to discrepancies that might arise from artifacts introduced by chemical fixation. It has been proposed that HPF fixation of cells provides a more reliable means to study vesicle docking via EM (Hammarlund et al., 2007, 2008), so we performed EM using both fixation methods.

Using chemical fixation, we did not observe a significant change in vesicle docking after cleavage of syb. In contrast, samples prepared using HPF fixation confirmed a loss in the number of docked vesicles, with no other change in the distribution of vesicles within $330 \mathrm{~nm}$ of the plasma membrane (Fig. S2).

To confirm that the observed defects in docking resulted from cleavage of syb, a toxin-resistant mutant form of the protein (syb-AA), in which two residues flanking the toxin cleavage site were substituted with alanines (K59A and L60A), was coexpressed with BoNT/D-LC in cells (Fig. 1 C). syb-AA largely restored the loss in secretion that occurs upon cleavage of endogenous syb (syb-AA, $15.3 \pm 5.5 / \mathrm{min} ; \mathrm{P}<0.001$, compared with the BoNT/D group; P > 0.05, compared with control cells lacking BoNT/D and syb-AA; Fig. 1 D); the slight loss of function during exocytosis is consistent with a previous study (Han and Jackson, 2006). Importantly, the docking defect caused by BoNT/D-LC was also fully rescued by syb-AA (syb-AA, $0.59 \pm 0.04 / \mu \mathrm{m}^{2} ; \mathrm{P}<0.001$, compared with the BoNT/D group; $\mathrm{P}>0.05$, compared with control cells; Fig. 1, G-I). As the defects in LDCV docking, a result of the cleavage of syb, were not secondary to changes in the total number of vesicles per cell (Fig. S3, A-C) or to the cotransfection of tPA-GFP with BoNT/D (Fig. S3, D-I), we conclude that syb indeed plays a role in the docking of LDCVs in PC12 cells.

As alluded to above, the SV protein syt-I has also been implicated in docking by interacting with the t-SNAREs syx-1A 
Figure 2. Defects in vesicle docking caused by cleavage of syb are not rescued by overexpression of syt-I, SNAP-25B, or syx-1A. (A-E) Typical TIRF images of LDCV docking in wild-type PC12 cells, cells expressing BoNT/ D-LC, and cells expressing BoNT/D-LC plus syt-l, SNAP-25B, or syx-1A. Bars, $5 \mu \mathrm{m}$. (F) The normalized cumulative distribution of docked LDCVs in $\mathrm{PC} 12$ cells was unaffected by overexpression of syt-l, SNAP-25B, or syx-1A. ( $G$ and $H$ ) Bar graphs showing that the density of docked vesicles $(G)$ and the rate of vesicle fusion $(H)$ were unaffected by syt-l (docking, $n=62$; fusion, $n=4$ ), SNAP-25B (docking, $n=63$; fusion, $n=5)$, or syx-1A overexpression (docking, $n=56$; fusion, $n=4$ ), as compared with cells expressing BoNT/D-LC alone (docking, $n=59$; fusion, $n=6$ ). Wild-type cells: docking, $n=68$; fusion, $n=3$. ${ }^{* *}, \mathrm{P}<0.001$. Error bars represent SEM.

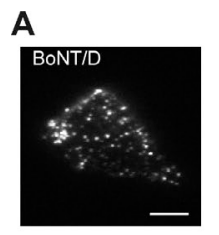

B

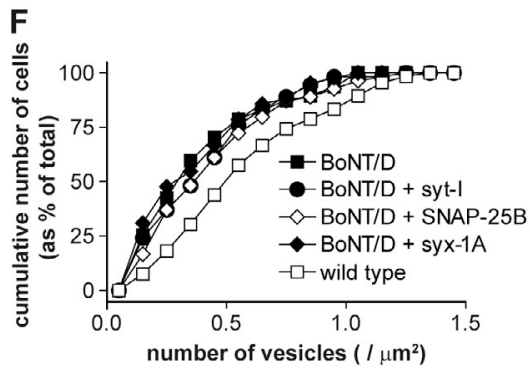

C
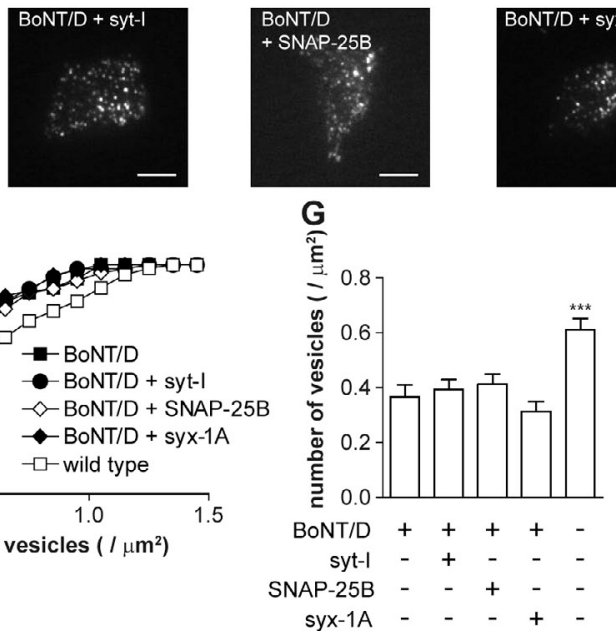

E

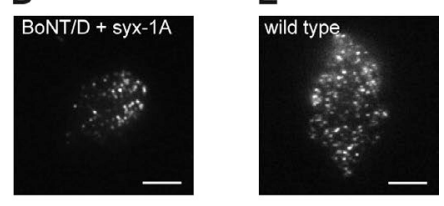

$\mathrm{H}$

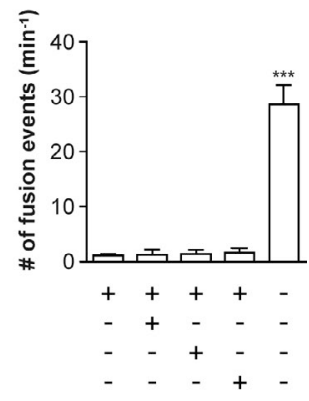

and SNAP-25B (Reist et al., 1998; de Wit et al., 2009). So, one explanation for the observed docking defects induced by syb cleavage is that syb might facilitate the interaction between syt-I and t-SNAREs and thus indirectly promote docking of LDCVs. To address this, we monitored LDCV docking in cells that overexpressed syt-I, syx-1A, or SNAP-25B along with the LC of BoNT/D (Fig. 2, A-E). However, we found that overexpression of these proteins failed to rescue the defects in docking $\left(\mathrm{BoNT} / \mathrm{D}, 0.37 \pm 0.04 / \mu^{2}\right.$; syt-I, $0.39 \pm 0.04 / \mu \mathrm{m}^{2}$; SNAP-25B, $0.41 \pm 0.04 / \mu \mathrm{m}^{2} ;$ syx-1A, $0.31 \pm 0.04 / \mu \mathrm{m}^{2} ; \mathrm{P}>0.05$, compared with BoNT/D-expressing cells; wild-type cells, $0.61 \pm 0.04 / \mu^{2}$; $\mathrm{P}<0.001$, compared with BoNT/D-expressing cells; Fig. 2, $\mathrm{F}$ and $\mathrm{G}$ ) or fusion (BoNT/D, $1.0 \pm 0.4 / \mathrm{min}$; syt-I, $1.3 \pm 0.9 / \mathrm{min}$; SNAP-25B, $1.4 \pm 0.7 / \mathrm{min}$; syx-1A, $1.5 \pm 0.9 / \mathrm{min}$; P > 0.05, compared with BoNT/D-expressing cells; wild-type cells, $28.7 \pm$ 3.5/min; P < 0.001, compared with BoNT/D-expressing cells; Fig. $2 \mathrm{H}$ ) that resulted from the cleavage of syb. Together, these results support a model in which syb directly participates in LDCV docking in PC12 cells.

\section{The membrane-distal region of the SNARE} motif of syb is vital for LDCV docking

Next, we determined whether the SNARE motif of syb (residues 31-85) is important for vesicle attachment to the plasma membrane. To this end, we generated a series of soluble, C-terminaltruncated syb constructs that contained different portions of its SNARE motif. One mutant contained the membrane-distal half of the motif (residues 1-52, designated as syb1-52), whereas the other two either constructs either fully excluded this motif (residues 1-30, designated as syb1-30) or included the entire motif (i.e., the entire cytoplasmic domain of syb, residues 1-96, designated as cd-syb; Fig. 3 A). Each construct was expressed in wild-type PC12 cells, and the impact on docking, mediated by endogenous syb, was evaluated (Fig.3, B-E).

cd-syb strongly inhibited docking (control, $0.66 \pm 0.04 / \mu^{2}$; cd-syb, $0.25 \pm 0.03 / \mu \mathrm{m}^{2}$; P $<0.001$; Fig. 3, C, F, and G) and virtually abolished fusion (control, $32.0 \pm 5.0 / \mathrm{min}$; cd-syb, $1.1 \pm$ $0.6 / \mathrm{min}$; $\mathrm{P}<0.001$; Fig. $3 \mathrm{H}$ ) of vesicles; this latter finding is consistent with a previous study (Hua and Scheller, 2001).
Thus, in addition to competitively binding to t-SNAREs to eliminate vesicle exocytosis, cd-syb also interacts with the docking partner of syb on the plasma membrane to prevent vesicle attachment. Interestingly, syb1-52 induced similar defects in the docking and fusion of LDCVs (syb1-52, $0.27 \pm$ $0.03 / \mu \mathrm{m}^{2}, 1.3 \pm 0.4 / \mathrm{min} ; \mathrm{P}<0.001$, compared with control; $\mathrm{P}>0.05$, compared with cells expressing cd-syb; Fig. 3, D and F-H), whereas syb1-30 had no effect (syb1-30, $0.58 \pm 0.04 / \mu \mathrm{m}^{2}$, $28.1 \pm 4.4 / \mathrm{min} ; \mathrm{P}>0.05$, compared with control; Fig. 3, E-H). These findings indicate that the N-terminal part of its SNARE motif is essential for syb to function in docking.

The membrane-proximal region of the SNARE motif of syb is crucial for fusion, but not docking, of LDCVs

It has been proposed that membrane fusion is mediated by the progressive assembly, or zippering, of the SNARE complex from the membrane-distal $\mathrm{N}$ termini of SNARE proteins toward the C-terminal membrane anchors of syb and syx. Therefore, we sought to determine whether this zipper model is applicable to syb-mediated vesicle docking. To test this idea, we generated a deletion within syb-AA, in which 16 residues (aa 67-82) were removed from the membrane-proximal C-terminal half of the SNARE motif (designated as syb $\Delta 67-82$; Fig. 3 I). Loss of vesicle docking as a result of cleavage of syb (Fig. 3, $\mathrm{J}$ and $\mathrm{K}$ ) was largely rescued by coexpressing the syb $\Delta 67-82$ truncation mutant along with BoNT/D (control, $0.66 \pm 0.04 / \mu^{2}$; BoNT/D, $0.31 \pm 0.03 / \mu m^{2} ;$ BoNT/D + syb $\Delta 67-82,0.52 \pm$ $0.04 / \mu \mathrm{m}^{2} ; \mathrm{P}<0.001$, compared with cells expressing BoNT/D; $\mathrm{P}<0.05$, compared with control; Fig. 3, L-N). However, this mutant failed to rescue fusion (control, $27.3 \pm 5.8 / \mathrm{min}$; BoNT/D, $0.8 \pm 0.4 / \mathrm{min} ; \mathrm{BoNT} / \mathrm{D}+$ syb $\Delta 67-82,1.0 \pm 0.4 / \mathrm{min} ; \mathrm{P}>0.05$, compared with cells expressing BoNT/D; Fig. 3 O). These results indicate that syb docks vesicles by binding to partnerdocking proteins on the target membrane through the N-terminal part of its SNARE motif but that subsequent fusion requires an intact C-terminal SNARE motif. These findings are in accordance with the zipper model for SNARE complex assembly, as detailed in the next section. 
A

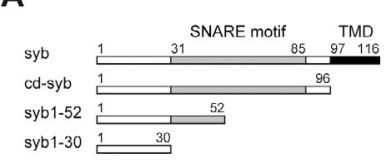

$\mathbf{F}$
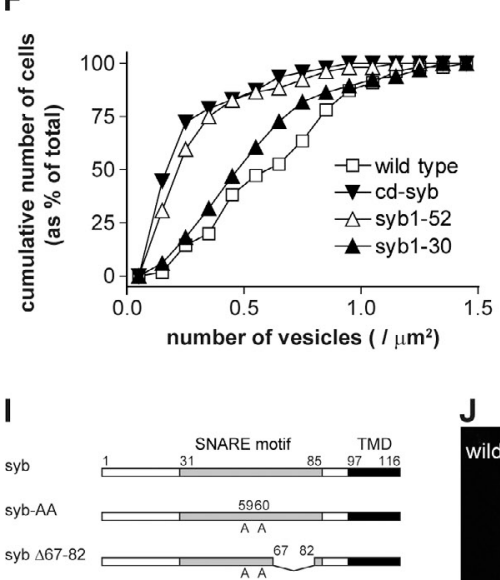

M

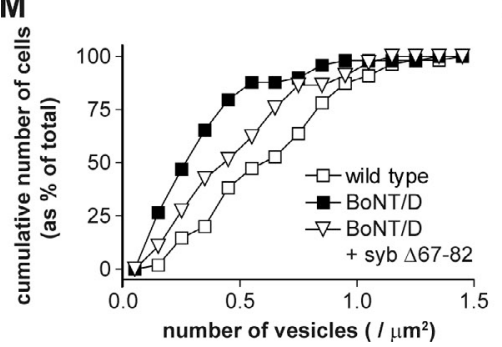

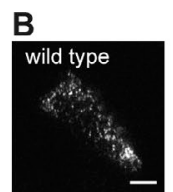

G

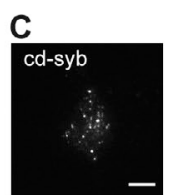

G

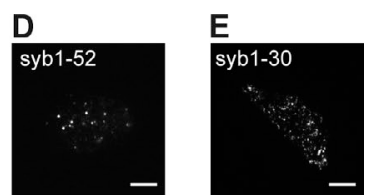

H
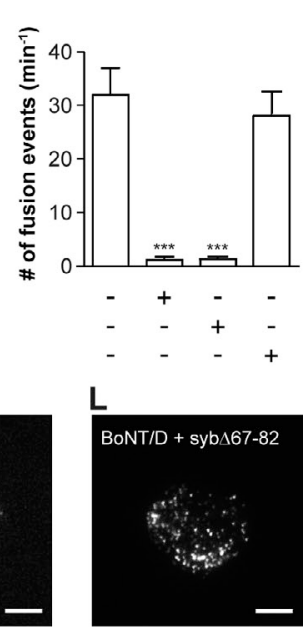

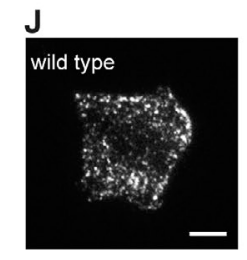

$\mathbf{N} \widetilde{\widetilde{\varepsilon}}$

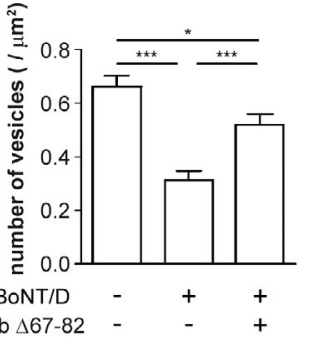

0

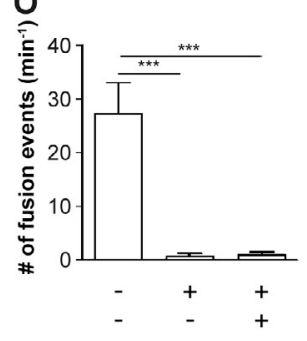

Figure 3. The membrane-distal portion of the SNARE motif is essential for syb-mediated docking of LDCVs in PC12 cells, whereas the membrane-proximal region is crucial for fusion but not docking. (A) Map of constructs of full-length and soluble $\mathrm{N}$-terminal fragments of syb. TMD, transmembrane domain. (B-E) Typical TIRF images of docked LDCVs in wild-type $\mathrm{PC} 12$ cells and cells expressing the indicated syb fragments. Bars, 5 um. (F) Normalized cumulative distribution of LDCVs showing that expression of syb 1-52 or cd-syb, but not sybl-30, induced defects in vesicle docking. ( $\mathrm{G}$ and $\mathrm{H}$ ) Bar graphs showing that the density of docked vesicles $(G)$ and the rate of vesicle fusion $(H)$ were strongly reduced by expression of syb 1 52 (docking, $n=69$; fusion, $n=6$ ) or cd-syb (docking, $n=62$; fusion, $n=7$ ) but remained unaffected by syb $1-30$ (docking, $n=68$; fusion, $n=7)$. Wild-type control cells: docking, $n=55$; fusion, $n=4$. (I) Map of constructs encoding full-length syb, the BoNT/D-resistant mutant syb-AA, and a syb mutant bearing a 16-aa deletion in the C-terminal portion of its SNARE motif, designated as syb $\Delta 67-82$. (J-L) Typical TIRF images of LDCV docking in wild-type PC 12 cells and cells expressing BoNT/D-LC with or without coexpression of syb $\Delta 67-82$. Bars, $5 \mu \mathrm{m}$. (M) Normalized cumulative distribution of LDCVs showing that expression of syb $\Delta 67-82$ rescued docking defects in cells that expressed BoNT/D-LC. (N and O) Bar graphs showing that syb $\Delta 67-82$ rescued defects in docking caused by cleavage of native syb $(\mathrm{N}$; control, $n=55 ;$ BoNT/D, $n=61$; BoNT/D + syb $\Delta 67-82$, $n=69$ ) but does not rescue inhibition of fusion (O; control, $n=4$; BoNT/D, $n=4$; BoNT/D + syb $\Delta 67-82, n=4) .(G, H, N$, and O) * $P<0.05$; $* * *, P<0.001$. Error bars represent SEM.
t-SNAREs are also critical for LDCV docking in live PC12 cells

Both t-SNAREs, syx-1A and SNAP-25B, have recently reemerged as crucial factors for the docking of LDCVs (de Wit et al., 2006, 2009; Hammarlund et al., 2007, 2008). Hence, t-SNAREs are strong candidates to serve as acceptors for syb-mediated vesicle docking in PC12 cells. To begin to test this idea, the LCs of BoNT/A or BoNT/C, which cleave SNAP-25B or SNAP-25B/syx-1A, respectively, were expressed in PC12 cells, and their effects on vesicle docking were evaluated by TIRFM. By immunostaining and immunoblot analysis, we confirmed that BoNT/A and BoNT/C efficiently cleave syx-1A and/or SNAP-25B in transfected cells (Fig. 4, A and B; Schiavo et al., 2000). As a result of SNAP-25B cleavage by BoNT/A or dual cleavage of SNAP-25B/syx-1A by BoNT/C (Blasi et al., 1993a,b; Schiavo et al., 1993), marked defects in vesicle docking were observed (control, $0.63 \pm 0.04 / \mu \mathrm{m}^{2}$; BoNT/A, $0.34 \pm$ $0.04 / \mu \mathrm{m}^{2} ;$ BoNT/C, $0.31 \pm 0.03 / \mu^{2} ; \mathrm{P}<0.001$, compared with control; Fig. 4, C and D), thus confirming recent EM studies and further validating the approach used here (de Wit et al., 2006, 2009). As expected, vesicle fusion was also eliminated by cleavage of t-SNAREs (control, $24.8 \pm 5.2 / \mathrm{min}$; BoNT/A, $1.5 \pm$ 0.6/min; BoNT/C, $1.1 \pm 0.4 / \mathrm{min} ; \mathrm{P}<0.001$, compared with control; Fig. 4 E; Schiavo et al., 2000). These results are consistent with a model in which t-SNAREs act as the plasma membrane partner proteins to dock LDCVs in PC12 cells.

\section{A model for LDCV docking}

The major finding in the current study is that syb plays a role in LDCV docking in PC12 cells (Figs. 1 and 2). We further show that a fragment of syb, which includes the membrane-distal half of the SNARE motif (syb1-52), inhibits native syb-mediated vesicle docking and fusion. In contrast, a C-terminal deletion mutant lacking 16 residues in the SNARE motif (syb $\Delta 67-82$ ) can still dock vesicles after full-length native syb has been removed by toxin cleavage (Fig. 3). As expected, syb $\Delta 67-82$ did not rescue vesicle fusion because this mutant cannot form completely zippered SNARE complexes. These results indicate that the N-terminal half of syb mediates vesicle docking.

We also confirmed the recent finding that the t-SNAREs syx and SNAP-25 also function as docking elements (Fig. 4; de Wit et al., 2006, 2009; Hammarlund et al., 2007, 2008). Thus, we speculate that after SNAP-25 and syx assemble into heterodimers on the plasma membrane, the $\mathrm{N}$ terminus of the SNARE motif of syb assembles with the membrane-distal SNARE motifs of the t-SNARE heterodimer to form partially assembled SNARE complexes to initiate docking. This model can be further tested using fluorescence resonance energy transfer experiments, performed via TIRFM, to determine whether docking is temporally associated with energy transfer between probes placed at the membrane-distal ends of $\mathrm{v}$ - and t-SNAREs. 
Figure 4. The t-SNAREs syx-1A and SNAP25B participate in LDCV docking reactions in PC12 cells. (A) Immunoblot analysis demonstrating cleavage of t-SNAREs by BoNT/A or BoNT/C. (left) Cleavage of SNAP-25 by BoNT/A or BoNT/C; the bottom band represents the larger of the two SNAP-25 cleavage products. (right) Cleavage of syx-1 by BoNT/ $\mathrm{C}-\mathrm{LC}$, as revealed by the reduction in the syx-1 band intensity; cleavage fragments were not observed. Transfection of BoNT-LCs resulted in an $\sim 30-40 \%$ reduction in the total amount of SNAP-25 or syx-1, which is in line with the observed $\sim 30 \%$ transfection efficiency. (B) Immunocytochemistry demonstrating cleavage of SNAP-25 (right column) by expression of BoNT/A-LC; the antibody used for imaging, SNAP-25-C, recognizes only the toxin-cleaved form of the protein. Cleaved SNAP-25 was not detected in either control group (tPA-GFP only, left column; tPA-GFP plus empty DsRed vector, middle column). Bar, 5 mm. (C) Normalized cumulative distribution of LDCVs showing that expression of BoNT/A-LC or BoNT/C-LC results in docking defects. ( $D$ and $E$ ) Bar graphs showing that the density of docked vesicles (D) and the rate of vesicle fusion (E) were markedly reduced by cleavage of t-SNAREs by BoNT/A-LC (docking, $n=62$; fusion, $n=4$ ) or BoNT/C-LC (docking, $n=71$; fusion, $n=4$ ) compared with wild-type control cells (docking, $n=69$; fusion, $n=4) .{ }^{* *}, P<0.001$. Error bars represent SEM.
A

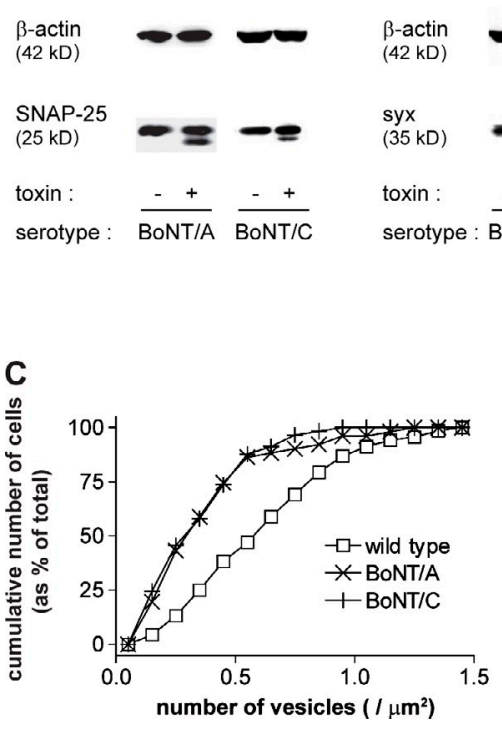

B

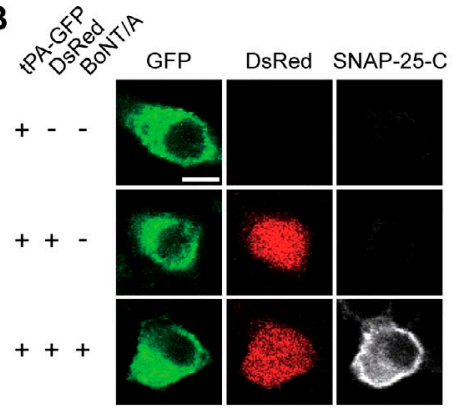

D

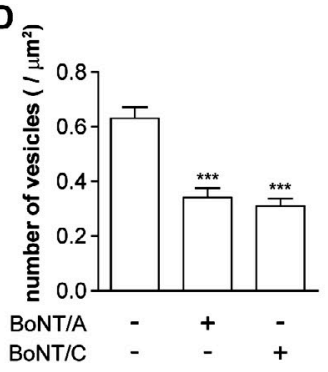

E

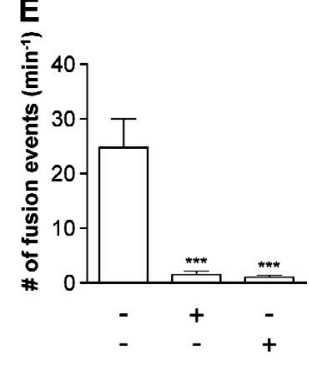

\section{Materials and methods}

\section{Cell culture}

PC12 cells were cultured in 10-cm dishes in DME (Corning) supplemented with $5 \%$ equine serum and $5 \% \mathrm{BCS}$ at $37^{\circ} \mathrm{C}$ with $10 \% \mathrm{CO}_{2}$. For imaging, cells were transferred to a \#1.5-thickness 25 -mm round coverslip (Warner Instruments) coated with collagen-I (BD) and polyD-lysine (Sigma-Aldrich).

Constructs, transfection, immunocytochemistry, and immunoblot analysis Transient transfection of tPA-GFP (a gift from B. Scalettar, Lewis \& Clark College, Portland, OR; Silverman et al., 2005), the LCs of BoNT/A and BoNT/C (pSyn-Lox-DsRed; Dong et al., 2006; Gascón et al., 2008), fulllength syt-1, syx-1A, SNAP-25B, syb-AA, syb $\Delta 67-82$ (all in pcDNA 3.1), and syb truncation mutants (pIRES-DsRed) into PC 12 cells was performed using Lipofectamine 2000 (Invitrogen) according to the manufacturer's protocol. cDNA for syb, SNAP-25B, and syx-1A was provided by J.E. Rothman (Yale University, New Haven, CT). cDNA encoding syt-l was provided by T.C. Sudhof (Stanford School of Medicine, Stanford, CA; note that the D374 mutation was corrected by substitution with a glycine residue; Desai et al., 2000). cDNA encoding the LCs of BoNT/A, C, and D was synthesized by GenScript. The LC of BoNT/D was selected because it cleaves both syb I and II from rat (Yamasaki et al., 1994); PC12 cells are derived from rats and express both syb isoforms (Chilcote et al., 1995). Mouse monoclonal antibodies specific for syb II (Cl 69.1), syx-1A (HPC 1), and SNAP-25 (Cl 71.2), provided by R. Jahn (Max Planck Institute for Biophysical Chemistry, Göttingen, Germany), were used to detect SNAREs. A polyclonal chicken anti-GFP antibody (Abcam) was used to label transfected cells. To monitor the cleavage of SNAP-25 via immunocytochemistry, we used an antibody that recognizes only the BoNT/Acleaved form of the protein $(\mathrm{Cl} 4 \mathrm{~F} 3-2 \mathrm{Cl}$; Research and Diagnostic Antibodies). For immunostaining, Cy2-conjugated anti-chicken (Jackson ImmunoResearch Laboratories, Inc.) and Alexa Fluor 647-conjugated anti-mouse (Invitrogen) secondary antibodies (1:500) were used. Images were collected using a confocal microscope (FV1000; Olympus) with a $60 \times 1.10 \mathrm{NA}$ water immersion objective and were acquired using FluoView software (version 1.6; Olympus). For 3D reconstructions, $z$-series images of $\mathrm{PC} 12$ cells were collected at depth increments of $0.5 \mu \mathrm{m}$. $Z$ projections were summed from $z$ stacks using ImageJ (version 1.42; National Institutes of Health).
For immunoblotting, PC 12 cells were collected using $100 \mu$ per well (24-well plate) of lysis buffer (PBS with 1\% Triton X-100, and $0.05 \%$ SDS plus a protease inhibitor cocktail [Roche]). Lysates were centrifuged for $10 \mathrm{~min}$ in a microcentrifuge at $4^{\circ} \mathrm{C}$, and the supernatants were subjected to SDS-PAGE and immunoblot analysis; immunoreactive bands were visualized using $\mathrm{ECL}$.

For EM, PC1 2 cells were cotransfected with two vectors, pIRESDsRed and pcDNA3.1 (with or without the BoNT/D-LC CDNA) at a ratio of $1: 2$, using Lipofectamine 2000. Cells that expressed DsRed were isolated using a customized flow cytometer (SORP FACSAria; BD). Efficient cleavage of syb in cells with red fluorescence was confirmed via immunocytochemistry.

EM

For chemical fixation, sorted cells were plated on coverslips coated with collagen-I and poly-D-lysine. The samples were fixed in 2.5\% (volume/ volume) glutaraldehyde and $2.0 \%$ (volume/volume) formaldehyde in $0.1 \mathrm{M}$ cacodylate buffer, $\mathrm{pH} \mathrm{7.4,} \mathrm{for} 45 \mathrm{~min}$ at room temperature and postfixed in $2.0 \%$ (weight/volume) osmium tetroxide in $0.1 \mathrm{M}$ sodium phosphate buffer at $22^{\circ} \mathrm{C}$ for $1 \mathrm{~h}$. The cells were dehydrated in a graded concentration series of ethanol at $22^{\circ} \mathrm{C}(50-100 \%$, volume/volume). Propylene oxide was used as the transition solvent. The dehydrated cells were infiltrated and embedded using ACM Fluka resin (Durcupam; Sigma-Aldrich). Final embedding was performed at $65^{\circ} \mathrm{C}$ for $24 \mathrm{~h}$. After polymerization, the glass coverslips were etched away using $50 \%$ hydrofluoric acid, and samples were rinsed in water. Ultrathin sections $(70-80 \mathrm{~nm})$ were generated using an ultramicrotome (EMUC6; Leica), placed on 300 mesh $\mathrm{Cu}$ Gilder thin-bar grids, poststained in uranyl acetate and Reynold's lead citrate, and viewed at $80 \mathrm{kV}$ on a transmission electron microscope (Philips CM120; FEI Corp.). Digital micrographs were taken using a digital camera (SIS MegaView III; Olympus)

For HPF, sorted cells were plated on sapphire discs coated with collagen-l and poly-D-lysine. Discs with cells were transferred to aluminum freezing planchettes (Wohlwend $\mathrm{GmbH}$ ) containing a layer of culture media plus 7\% Ficoll PM 70 (Sigma-Aldrich) and subjected to HPF in HPMO1O (Leica). Frozen discs/cells were transferred to cryotubes (Thermo Fisher Scientific) containing freeze substitution media and cooled to $-90^{\circ} \mathrm{C}$ using an automatic freeze substitution system (Leica). Freeze substitution was performed as previously described (Morphew and Mclntosh, 2003). In brief, samples were placed in $0.5 \%$ glutaraldehyde (Electron Microscopy Services) and 0.1\% tannic acid (Mallinckrodt, Inc.) in acetone; 
substitution was performed by placing samples in a bath containing $1 \% \mathrm{OsO}_{4}$ (Electron Microscopy Services) and $0.1 \%$ uranyl acetate (Electron Microscopy Services) in acetone. Samples were then warmed to $0^{\circ} \mathrm{C}$, rinsed in acetone, infiltrated with epoxy resin (EPOX-Araldite; Electron Microscopy Services), and sapphire discs were flat embedded between two microscope slides that were treated with Teflon-like spray (Electron Microscopy Services; Reymond and Pickett-Heaps, 1983). After polymerization of the resin, the sapphire disc was released from the epoxy, and designated cells were mounted and sectioned en face. 80-nm serial sections were cut using a microtome (Ultracut; Leica), collected on formvarcoated copper slot grids, and poststained with uranyl acetate and lead citrate. Grids were examined on an electron microscope (Philips CM10) operating at $80 \mathrm{kV}$, and images were recorded using a V602 camera (Advanced Microscopy Techniques).

LDCVs were identified by their round, dense core and had a diameter of $\sim 90 \mathrm{~nm}(\sim 70-120 \mathrm{~nm})$. Analysis of the LDCV distribution was performed blind, and only vesicles directly touching the plasma membrane were scored as docked.

\section{TIRFM and analysis of acquired images}

PC 12 cell images were acquired in an incubation buffer $150 \mathrm{mM} \mathrm{NaCl}$, $4.2 \mathrm{mM} \mathrm{KCl}, 1 \mathrm{mM} \mathrm{NaH}{ }_{2} \mathrm{PO}_{4}, 0.7 \mathrm{mM} \mathrm{MgCl}, 2 \mathrm{mM} \mathrm{CaCl}$, and $10 \mathrm{mM}$ Hepes, pH 7.4) at room temperature using a microscope (IX81-ZDC; Olympus) configured for evanescent field excitation and equipped with a $1.45 \mathrm{NA}$ objective (Apo 100x and Apo 60x; Olympus) and an electron-multiplying charge-coupled device camera (C9100-02; Hamamatsu Photonics) with an image pixel size of $80 \mathrm{~nm}$. Image acquisition was controlled using SlideBook software (version 5.0; Olympus) at a rate of $2 \mathrm{~Hz}$, with an exposure time of $100 \mathrm{~ms}$ for each frame. The excitation wavelengths were $488 \mathrm{~nm}$ for GFP and $543 \mathrm{~nm}$ for DsRed. The incident angle of the excitation light beam was $\sim 70^{\circ}$, which was determined using a prism. The decay constant was calculated to be $\sim 110 \mathrm{~nm}$, using a refractive index of 1.37 (Axelrod, 1981). For analysis based on static images, densities of LDCVs from TIRF images were analyzed using MetaMorph software (version 7.0; Molecular Devices) and ImageJ (version 1.42). For analysis of vesicle dynamics, vesicles were detected and tracked using the spots function in iMaris software (Bitplane).

In TIRFM experiments, cells for analysis were indentified by strong green (GFP) and/or red (DsRed) fluorescence in the widefield mode of the TIRF microscope. Cells with DsRed fluorescence always showed a strong GFP signal because GFP (tPA construct) expression/fluorescence was better than DsRed (toxin constructs). Green fluorescence images were taken in both the widefield and TIRF modes, and red fluorescence images were taken in the widefield mode only. Transfected cells with normal morphology and clearly delineated green puncta were selected for analysis, and no more than 10 cells were selected from each coverslip. Bright cells were selected at random in each group to avoid bias; $\sim 60-70$ cells were analyzed for each experimental condition.

We defined immobile, docked vesicles as green puncta that stay within the evanescent field (in Z) for $5 \mathrm{~s}$ within a 2-pixel displacement (in X-Y): $\sim 80 \%$ of the vesicles were immobile in the control, and $\sim 70 \%$ of the vesicles were immobile in the presence of the BoNT/D-LC in PC12 cells. As most of the vesicles were immobile, only static analysis was performed in the following experiments. We developed a program in MATLAB (MathWorks) for semiautomated detection of vesicles, which applied an algorithm of an à trous wavelet transformation to individual images. This transformation was shown to be effective in extracting spots and removing noise in biological images (Olivo-Marin, 2002) and has been used in a previous study to analyze TIRF images (Toonen et al., 2006). In brief, the à trous wavelet transform decomposes the image into subband images corresponding to different spatial resolutions by convolution with kernels at various scales. For a spot that is small globally but relatively large locally on a pixel basis and with a large intensity discontinuity compared with the adjacent background, the presence of the spot affects multiple scales of the wavelet subband images from the à trous transform. Local Gaussian noise is averaged across larger-scale wavelet images; only the smallest scale images are affected. By correlating the wavelet coefficient at multiple scales, features contributed in multiple scales are amplified, whereas random Gaussian noise is suppressed. An intensity threshold is first used to convert each wavelet image into a binary mask that is nonzero only at positions with prominent coefficients. The threshold was set with an iteration level of $k=3$ and a detection threshold of $I_{d}=1$, according to Toonen et al. (2006). The resulting correlated image represents detected vesicles as puncta. Only puncta with an area $>4$ pixels were counted. Docking density was calculated by dividing the number of docked vesicles by the area of the cell footprint.
In addition to the à trous wavelet transformation approach, we also applied a simple method to measure docking. Again, bright cells were selected in widefield mode. Then, in TIRF mode, the brightest $\sim 10$ vesicles from each cell (again, no more than 10 cells per coverslip) were selected. For each coverslip, the mean intensities of the brightest vesicles were calculated, and $5 \%$ of this mean was used as the threshold; puncta $>6$ pixels were counted. Results obtained from this manual analysis closely agreed with the findings obtained using the à trous wavelet transformation, so only the latter is reported.

To stimulate exocytosis, individual cells were depolarized via superfusion with a high [KCl] buffer $105 \mathrm{mM} \mathrm{KCl}, 50 \mathrm{mM} \mathrm{NaCl}, 2 \mathrm{mM}$ $\mathrm{CaCl}_{2}, 0.7 \mathrm{mM} \mathrm{MgCl}_{2}, 1 \mathrm{mM} \mathrm{NaH} \mathrm{PO}_{4}$, and $10 \mathrm{mM}$ Hepes, $\mathrm{pH}$ 7.4). Fusion events were detected as a transient increase in green fluorescence signal (tPA-GFP) followed by diffusion away from the site of exocytosis (Zhang et al., 2011 ). Image acquisition rate was $2 \mathrm{~Hz}$ with a 100-ms exposure time for $60 \mathrm{~s}$.

Statistical analysis

Data are shown as mean values \pm SEM. The $p$-values used for multiple comparisons were calculated using one-way analysis of variance with Bonferroni correction (GraphPad Prism 4; GraphPad Software).

\section{Online supplemental material}

Fig. S1 shows the distribution of docked vesicle density was similar among different experiments. Fig. S2 shows a comparison of docking results obtained using $\mathrm{EM}$ of $\mathrm{PC} 12$ cells prepared using either chemical fixation or the HPF method. Fig. S3 shows that the total number of LDCVs in PC1 2 cells was unaffected by cleavage of SNARE proteins, and LDCV docking was unaffected by cotransfection protocols. Online supplemental material is available at http://www.jcb.org/cgi/content/full/jcb.201106158/DC1

We thank M.B. Jackson, J. Audhya, and X. Lou for critical comments regarding this manuscript, A. Hoenger and R. Massey for help with EM, J. Campbell for carrying out HPF, and M. Zhao for help with image processing.

This work was supported by grants from the National Institutes of Health (MH 61876). M.K. Morphew is supported by a National Institutes of Health grant (8P4 1GM103431-42). J. Yao is supported by an American Heart Association postdoctoral fellowship (1 1 POST57200 16). E.R. Chapman is an Investigator of the Howard Hughes Medical Institute.

Submitted: 28 June 2011

Accepted: 28 June 2012

\section{References}

Axelrod, D. 1981. Cell-substrate contacts illuminated by total internal reflection fluorescence. J. Cell Biol. 89:141-145. http://dx.doi.org/10.1083/jcb.89.1.141

Axelrod, D. 2001. Total internal reflection fluorescence microscopy in cell biology. Traffic. 2:764-774. http://dx.doi.org/10.1034/j.1600-0854.2001.21104.x

Blasi, J., E.R. Chapman, E. Link, T. Binz, S. Yamasaki, P. De Camilli, T.C. Südhof, H. Niemann, and R. Jahn. 1993a. Botulinum neurotoxin A selectively cleaves the synaptic protein SNAP-25. Nature. 365:160-163. http:// dx.doi.org/10.1038/365160a0

Blasi, J., E.R. Chapman, S. Yamasaki, T. Binz, H. Niemann, and R. Jahn. 1993b. Botulinum neurotoxin $\mathrm{C} 1$ blocks neurotransmitter release by means of cleaving HPC-1/syntaxin. EMBO J. 12:4821-4828.

Borisovska, M., Y. Zhao, Y. Tsytsyura, N. Glyvuk, S. Takamori, U. Matti, J. Rettig, T. Südhof, and D. Bruns. 2005. v-SNAREs control exocytosis of vesicles from priming to fusion. EMBO J. 24:2114-2126. http://dx.doi .org/10.1038/sj.emboj.7600696

Broadie, K., A. Prokop, H.J. Bellen, C.J. O'Kane, K.L. Schulze, and S.T. Sweeney. 1995. Syntaxin and synaptobrevin function downstream of vesicle docking in Drosophila. Neuron. 15:663-673. http://dx.doi.org/ 10.1016/0896-6273(95)90154-X

Chapman, E.R. 2008. How does synaptotagmin trigger neurotransmitter release? Annu. Rev. Biochem. 77:615-641. http://dx.doi.org/10.1146/annurev .biochem.77.062005.101135

Chilcote, T.J., T. Galli, O. Mundigl, L. Edelmann, P.S. McPherson, K. Takei, and P. De Camilli. 1995. Cellubrevin and synaptobrevins: Similar subcellular localization and biochemical properties in PC12 cells. J. Cell Biol. 129:219-231. http://dx.doi.org/10.1083/jcb.129.1.219

Deák, F., S. Schoch, X. Liu, T.C. Südhof, and E.T. Kavalali. 2004. Synaptobrevin is essential for fast synaptic-vesicle endocytosis. Nat. Cell Biol. 6:1102-1108. http://dx.doi.org/10.1038/ncb1185 
de Wit, H., L.N. Cornelisse, R.F. Toonen, and M. Verhage. 2006. Docking of secretory vesicles is syntaxin dependent. PLOS ONE. 1:e126. http://dx.doi .org/10.1371/journal.pone.0000126

de Wit, H., A.M. Walter, I. Milosevic, A. Gulyás-Kovács, D. Riedel, J.B. Sørensen, and M. Verhage. 2009. Synaptotagmin-1 docks secretory vesicles to syntaxin-1/SNAP-25 acceptor complexes. Cell. 138:935-946. http://dx.doi .org/10.1016/j.cell.2009.07.027

Desai, R.C., B. Vyas, C.A. Earles, J.T. Littleton, J.A. Kowalchyck, T.F. Martin, and E.R. Chapman. 2000. The C2B domain of synaptotagmin is a $\mathrm{Ca}^{2+}$ sensing module essential for exocytosis. J. Cell Biol. 150:1125-1136. http://dx.doi.org/10.1083/jcb.150.5.1125

Dong, M., F. Yeh, W.H. Tepp, C. Dean, E.A. Johnson, R. Janz, and E.R. Chapman. 2006. SV2 is the protein receptor for botulinum neurotoxin A. Science. 312:592-596. http://dx.doi.org/10.1126/science.1123654

Fernández-Busnadiego, R., B. Zuber, U.E. Maurer, M. Cyrklaff, W. Baumeister, and V. Lucic. 2010. Quantitative analysis of the native presynaptic cytomatrix by cryoelectron tomography. J. Cell Biol. 188:145-156. http:// dx.doi.org/10.1083/jcb.200908082

Gascón, S., J.A. Paez-Gomez, M. Díaz-Guerra, P. Scheiffele, and F.G. Scholl. 2008. Dual-promoter lentiviral vectors for constitutive and regulated gene expression in neurons. J. Neurosci. Methods. 168:104-112. http://dx.doi .org/10.1016/j.jneumeth.2007.09.023

Geppert, M., Y. Goda, R.E. Hammer, C. Li, T.W. Rosahl, C.F. Stevens, and T.C. Südhof. 1994. Synaptotagmin I: A major Ca2+ sensor for transmitter release at a central synapse. Cell. 79:717-727. http://dx.doi.org/ 10.1016/0092-8674(94)90556-8

Gerber, S.H., J.C. Rah, S.W. Min, X. Liu, H. de Wit, I. Dulubova, A.C. Meyer, J. Rizo, M. Arancillo, R.E. Hammer, et al. 2008. Conformational switch of syntaxin-1 controls synaptic vesicle fusion. Science. 321:1507-1510. http://dx.doi.org/10.1126/science.1163174

Hammarlund, M., M.T. Palfreyman, S. Watanabe, S. Olsen, and E.M. Jorgensen. 2007. Open syntaxin docks synaptic vesicles. PLoS Biol. 5:e198. http://dx.doi .org/10.1371/journal.pbio.0050198

Hammarlund, M., S. Watanabe, K. Schuske, and E.M. Jorgensen. 2008. CAPS and syntaxin dock dense core vesicles to the plasma membrane in neurons. J. Cell Biol. 180:483-491. http://dx.doi.org/10.1083/jcb.200708018

Han, X., and M.B. Jackson. 2006. Structural transitions in the synaptic SNARE complex during $\mathrm{Ca}^{2+}$-triggered exocytosis. J. Cell Biol. 172:281-293. http://dx.doi.org/10.1083/jcb.200510012

Hua, Y., and R.H. Scheller. 2001. Three SNARE complexes cooperate to mediate membrane fusion. Proc. Natl. Acad. Sci. USA. 98:8065-8070. http:// dx.doi.org/10.1073/pnas.131214798

Hunt, J.M., K. Bommert, M.P. Charlton, A. Kistner, E. Habermann, G.J. Augustine, and H. Betz. 1994. A post-docking role for synaptobrevin in synaptic vesicle fusion. Neuron. 12:1269-1279. http://dx.doi.org/ 10.1016/0896-6273(94)90443-X

Liu, H., C. Dean, C.P. Arthur, M. Dong, and E.R. Chapman. 2009. Autapses and networks of hippocampal neurons exhibit distinct synaptic transmission phenotypes in the absence of synaptotagmin I. J. Neurosci. 29:7395-7403. http://dx.doi.org/10.1523/JNEUROSCI.1341-09.2009

Morphew, M.K., and J.R. McIntosh. 2003. The use of filter membranes for highpressure freezing of cell monolayers. J. Microsc. 212:21-25. http://dx.doi org/10.1046/j.1365-2818.2003.01231.x

O'Connor, V., C. Heuss, W.M. De Bello, T. Dresbach, M.P. Charlton, J.H. Hunt, L.L. Pellegrini, A. Hodel, M.M. Burger, H. Betz, et al. 1997. Disruption of syntaxin-mediated protein interactions blocks neurotransmitter secretion. Proc. Natl. Acad. Sci. USA. 94:12186-12191. http://dx.doi.org/ 10.1073/pnas.94.22.12186

Olivo-Marin, J.C. 2002. Extraction of spots in biological images using multiscale products. Pattern Recognition. 35:1989-1996. http://dx.doi.org/10.1016/ S0031-3203(01)00127-3

Reist, N.E., J. Buchanan, J. Li, A. DiAntonio, E.M. Buxton, and T.L. Schwarz. 1998. Morphologically docked synaptic vesicles are reduced in synaptotagmin mutants of Drosophila. J. Neurosci. 18:7662-7673.

Reymond, O.L., and J.D. Pickett-Heaps. 1983. A routine flat embedding method for electron microscopy of microorganisms allowing selection and precisely orientated sectioning of single cells by light microscopy. J. Microsc. 130:79-84. http://dx.doi.org/10.1111/j.1365-2818.1983.tb04200.x

Rothman, J.E. 1994. Intracellular membrane fusion. Adv. Second Messenger Phosphoprotein Res. 29:81-96. http://dx.doi.org/10.1016/S1040-7952 (06)80008-X

Schiavo, G., O. Rossetto, S. Catsicas, P. Polverino de Laureto, B.R. DasGupta, F. Benfenati, and C. Montecucco. 1993. Identification of the nerve terminal targets of botulinum neurotoxin serotypes A, D, and E. J. Biol. Chem. 268:23784-23787.

Schiavo, G., M. Matteoli, and C. Montecucco. 2000. Neurotoxins affecting neuroexocytosis. Physiol. Rev. 80:717-766.
Silverman, M.A., S. Johnson, D. Gurkins, M. Farmer, J.E. Lochner, P. Rosa, and B.A. Scalettar. 2005. Mechanisms of transport and exocytosis of densecore granules containing tissue plasminogen activator in developing hippocampal neurons. J. Neurosci. 25:3095-3106. http://dx.doi.org/10.1523/ JNEUROSCI.4694-04.2005

Söllner, T., M.K. Bennett, S.W. Whiteheart, R.H. Scheller, and J.E. Rothman. 1993. A protein assembly-disassembly pathway in vitro that may correspond to sequential steps of synaptic vesicle docking, activation, and fusion. Cell. 75:409-418. http://dx.doi.org/10.1016/0092-8674 (93) $90376-2$

Sørensen, J.B., G. Nagy, F. Varoqueaux, R.B. Nehring, N. Brose, M.C. Wilson, and E. Neher. 2003. Differential control of the releasable vesicle pools by SNAP-25 splice variants and SNAP-23. Cell. 114:75-86. http://dx.doi .org/10.1016/S0092-8674(03)00477-X

Sørensen, J.B., K. Wiederhold, E.M. Müller, I. Milosevic, G. Nagy, B.L. de Groot, H. Grubmüller, and D. Fasshauer. 2006. Sequential N- to C-terminal SNARE complex assembly drives priming and fusion of secretory vesicles. EMBO J. 25:955-966. http://dx.doi.org/10.1038/sj.emboj.7601003

Steyer, J.A., H. Horstmann, and W. Almers. 1997. Transport, docking and exocytosis of single secretory granules in live chromaffin cells. Nature. 388:474-478. http://dx.doi.org/10.1038/41329

Sutton, R.B., D. Fasshauer, R. Jahn, and A.T. Brunger. 1998. Crystal structure of a SNARE complex involved in synaptic exocytosis at $2.4 \mathrm{~A}$ resolution. Nature. 395:347-353. http://dx.doi.org/10.1038/26412

Toonen, R.F., O. Kochubey, H. de Wit, A. Gulyas-Kovacs, B. Konijnenburg, J.B. Sørensen, J. Klingauf, and M. Verhage. 2006. Dissecting docking and tethering of secretory vesicles at the target membrane. EMBO J. 25:3725-3737. http://dx.doi.org/10.1038/sj.emboj.7601256

Tsuboi, T., H.T. McMahon, and G.A. Rutter. 2004. Mechanisms of dense core vesicle recapture following "kiss and run" ("cavicapture") exocytosis in insulin-secreting cells. J. Biol. Chem. 279:47115-47124. http://dx.doi .org/10.1074/jbc.M408179200

Verhage, M., and J.B. Sørensen. 2008. Vesicle docking in regulated exocytosis. Traffic. 9:1414-1424. http://dx.doi.org/10.1111/j.1600-0854.2008.00759.x

Weber, T., B.V. Zemelman, J.A. McNew, B. Westermann, M. Gmachl, F. Parlati, T.H. Söllner, and J.E. Rothman. 1998. SNAREpins: minimal machinery for membrane fusion. Cell. 92:759-772. http://dx.doi.org/ 10.1016/S0092-8674(00)81404-X

Yamasaki, S., A. Baumeister, T. Binz, J. Blasi, E. Link, F. Cornille, B. Roques, E.M. Fykse, T.C. Südhof, R. Jahn, et al. 1994. Cleavage of members of the synaptobrevin/VAMP family by types D and F botulinal neurotoxins and tetanus toxin. J. Biol. Chem. 269:12764-12772.

Zenisek, D., J.A. Steyer, and W. Almers. 2000. Transport, capture and exocytosis of single synaptic vesicles at active zones. Nature. 406:849-854 http://dx.doi.org/10.1038/35022500

Zhang, Z., Y. Wu, Z. Wang, F.M. Dunning, J. Rehfuss, D. Ramanan, E.R. Chapman, and M.B. Jackson. 2011. Release mode of large and small dense-core vesicles specified by different synaptotagmin isoforms in PC12 cells. Mol. Biol. Cell. 22:2324-2336. http://dx.doi.org/10.1091/ mbc.E11-02-0159 